Man and Nature

L'homme et la nature

\title{
Rhétorique Féministe et Liberté Personnelle
}

\section{Olga B. Cragg}

Volume 8, 1989

URI : https://id.erudit.org/iderudit/1012602ar

DOI : https://doi.org/10.7202/1012602ar

Aller au sommaire du numéro

Éditeur(s)

Canadian Society for Eighteenth-Century Studies / Société canadienne d'étude du dix-huitième siècle

ISSN

0824-3298 (imprimé)

1927-8810 (numérique)

Découvrir la revue

Citer cet article

Cragg, O. B. (1989). Rhétorique Féministe et Liberté Personnelle. Man and Nature / L'homme et la nature, 8, 107-117. https://doi.org/10.7202/1012602ar

Copyright (C Canadian Society for Eighteenth-Century Studies / Sociéte canadienne d'étude du dix-huitième siècle, 1989
Ce document est protégé par la loi sur le droit d'auteur. L'utilisation des services d'Érudit (y compris la reproduction) est assujettie à sa politique d'utilisation que vous pouvez consulter en ligne.

https://apropos.erudit.org/fr/usagers/politique-dutilisation/ 


\section{Rhétorique Féministe et Liberté Personnelle}

Le terme anachronique, 'rhétorique féministe', ${ }^{1} \mathrm{du}$ titre de cette étude, porte sur plusieurs aspects révélateurs de l'univers imaginaire de Mme Riccoboni. L'oeuvre épistolaire de la romancière, ainsi que ses deux romans à la troisième personne, se distinguent par une rhétorique ${ }^{2}$ qui lui est propre et qui se manifeste par des énoncés de réduction généralisante. Concédons pourtant d'emblée, qu'au 18e siècle, définir l'homme, éclairer le public, l'instruire et le soumettre à l'examen de la raison deviennent une manie fort répandue chez les écrivains. Comme nous le savons, la vague moralisatrice s'accentue dans le domaine romanesque en France avec la popularité des romans de Richardson et de la Nouvelle Héloïse. Mme Riccoboni suit donc en un sens une route déjà tracée par ses illustres prédécesseurs. Cependant sa perspective sur la vie, marquée d'abord par son besoin d'abandonner sa carrière de comédienne et, ensuite, par sa réussite professionnelle d'écrivain se différencie de la vision morale de ses contemporains masculins, assez souvent teintée de préjugés misogynes. Les deux romans que nous allons discuter, séparés l'un de l'autre par une vingtaine d'années, reflètent chez l'auteur un changement subtil dans son traitement de la liberté féminine. En particulier, il sera intéressant d'examiner en détail l'Histoire de Monsieur le Marquis de Cressy (1758), ${ }^{3}$ qui appartient au début de la carrière littéraire de l'auteur, et de comparer ce roman à sa dernière oeuvre épistolaire, Lettres de Mylord Rivers (1777) pour essayer d'en dégager l'évolution idéologique consécutive à l'expérience personnelle de l'auteur.

Le féminisme qui s'annonce si clairement dans l'Histoire de Cressy et qui est reconnu par ses critiques comme faisant partie de l'univers spécifiquement riccobonien se lit à plusieurs niveaux. Non seulement le discours féminin s'affirme-t-il dans la structuration du récit où les événements parlent d'eux-mêmes, mais il est doublé des réflexions de l'auteur sur le sujet féminin. Le parti-pris de Mme Riccoboni dans son réquisitoire contre les hommes se trahit également par l'emploi d'un vocabulaire fortement coloré, dans la présentation et la description de ses personnages masculins. Nous voulons interroger surtout les conséquences de la pensée féministe de Mme Riccoboni sur le destin de ses deux héroïnes, Mme de Cressy et Adeline. Une évolution nette 
se manifeste dans la façon dont ces deux héroïnes perçoivent leur être et leurs sentiments par rapport à leur degré d'indépendance psychologique. La liberté de Mme de Cressy, si l'on peut qualifier d'un tel mot sa conduite, se révèle comme un souffle fragile et surtout négatif. Elle est prisonnière d'elle-même et des circonstances. En fait, telle est l'image de la femme répandue par la littérature féminine de l'époque. Par contre, presque une vingtaine d'années plus tard, l'invention d'un être féminin presque agressif se fait selon un modèle bien moins stéréotypé où l'énergie intérieure s'identifie avec la satisfaction des besoins personnels.

Sans approfondir un lien possible entre l'état d'âme de l'écrivain et la liberté intérieure ressentie au moment de la création, notons seulement quelques coïncidences entre les données biographiques et la conception de la femme proposée dans les deux romans en question. L'espace psychique de Mme Riccoboni, au moment de la genèse de $\mathrm{l}^{\prime}$ Histoire de Cressy, ne pouvait être que fort contraignant. Le joug marital pesa sur notre romancière jusqu'à la mort de son mari, quoiqu'ils ne partagent plus la même existence depuis 1756. La correspondance de Mme Riccoboni reflète fidèlement la tyrannie qu'elle a dû ressentir pendant cette période de séparation, de soi-disant indépendance. Du point de vue financier et émotionnel, elle a toujours été entravée par les exigences de son mari qui l'a dominée d'une façon subtile: 'Je l'ai obligé jusqu'au dernier instant, j'ai fait plus que je ne pouvais, ${ }^{4}$ se plaint-elle à David Garrick. Dans cette même lettre de 1772, elle parle de la mort de son mari comme d'un événement libérateur: 'J'ai soigneusement caché combien mon sort avoit de rigueur, et ceux qui me voyoient le plus souvent ne sçavoient pas la cause de mes chagrins et de la continuelle inquiétude qui souvent paroissoit malgré moi.' $\mathrm{Si}$ le repos et la liberté que Mme Riccoboni avait obtenus par cette disparition lui paraissaient quelquefois comme irréels, cette nouvelle indépendance devait indéniablement avoir quelque effet sur le développement de ses héroïnes féminines. Le contrôle de la destinée sur le plan affectif, qu'Adeline dans les Lettres de Mylord Rivers, découvre possible n'est pas sans quelque rapport avec la libération personnelle acquise par l'auteur elle-même.

L'image de l'homme que trace Mme Riccoboni dans l'Histoire de Cressy, quoique plus nuancée que celle des femmes, est plus sombre que sa représentation du même sexe dans son dernier roman, ce qui, en fait, n'a rien d'étonnant. Malgré quelques qualités flatteuses qui ornent le portrait formel de $\mathrm{M}$. de Cressy, ce sont les attributs masculins négatifs qui prédominent. Si l'on examine de près les composantes de sa nature, telles que cette miniature nous la révèle, ce qui saute aux yeux c'est l'importance attribuée à l'intelligence. Elle est primordiale, 
malgré son rôle néfaste, dans la liste des défauts: 'l'air doux, le coeur faux, beaucoup de finesse dans l'esprit, l'art de cacher ses vices, et de connoître le foible d'autrui, fondoient ses espérances' (p. 2). ${ }^{5}$ Or, $l^{\prime}$ hypocrisie, la dissimulation et la pénétration d'autrui sont toutes enracinées dans la raison. Ainsi l'intellect est conçu comme étant à la base de la personnalité masculine. Mais suivant la tradition moraliste classique, Mme Riccoboni se méfie du côté rationnel de l'homme, car elle $y$ voit la source de son orgueil et en fin de compte de sa chute.

Le féminisme de l'auteur dans l'Histoire de Cressy se révèle d'une manière éclatante lorsqu'elle sème à travers le texte des mots-clefs et des épithètes à valeur critique qui répartissent les personnages en bourreaux et en victimes. La dichotomie se fait sentir dès les premières pages du roman lorsque Mme Riccoboni se réfère à Adélaïde en l'appelant une 'triste victime,' (p. 4) tandis que le marquis est stigmatisé par sa 'perfidie' (p. 5). Le vocabulaire est ainsi chargé de valeurs affectives. Une touche de négativité est de plus injectée dans la narration quand des termes isolés ou des formules toutes faites, comme 'profiter d'un avantage,' 'affecter,' 'cacher cette ambition,' sont utilisées pour connoter les mobiles du marquis d'une façon peu flatteuse: de même, 'agir en apparence,' 'parvenu à se faire un mérite d'avoir trahi,' 's'applaudissoit de l'art avec lequel il la trompoit' - toutes ces expressions trahissent une réalité fortement subjectivisée. Il est manifeste que l'auteur 'omniscient' oriente la perspective de la narration dans un sens féministe lorsqu'elle attaque l'homme pour son insensibilité, sa duperie, son égoïsme et son aveuglement devant les vraies valeurs de l'existence et du bonheur. C'est l'âme féminine qui semble seule capable de détenir le secret du bonheur conjugal fondé sur le respect et l'estime mutuels.

D'autre part, l'architecture du roman traduit elle aussi la voix féminine par l'emploi d'un didactisme laconique. Nous avons repéré au moins quarante - trois locutions dans l'Histoire de Cressy où figure l'observation générale à tendance dogmatique et moralisante que les spécialistes de la parole proverbiale, prise dans son sens le plus large, désignent du terme technique de parémie. ${ }^{6}$ Dans l'Histoire de Cressy les interventions d'ordre général, extérieures au dialogue et à la narration des événements, appartiennent, pour la plupart, à la rhétorique externe par opposition à la rhétorique interne fonctionnelle employée par les personnages, comme chez Prévost par exemple, pour se convaincre les uns les autres. Précisons tout de suite que d'une part, ce regroupement de propositions didactiques comporte des observations psychologiques de valeur universelle qui découlent du contexte discursif, ou lui sont intégralement liées par la syntaxe grammaticale et ne peuvent pas en être séparées et que, d'autre part, on peut établir 
un inventaire assez riche, pour un roman aussi bref et concis que l'Histoire de Cressy, de segments isolables, qui ne sont autres que de véritables maximes. Par contre, lorsque la réflexion globale qui cherche à exprimer une idée essentialiste de la nature humaine ou à définir psychologiquement un certain comportement et à l'ériger en règle de conduite, se trouve incorporée dans le discours narratif, les observations abstraites et les maximes se confondent en un moralisme général. En effet, dans ce petit roman de Mme Riccoboni, nous avons pu distinguer trente-deux énoncés qui appartiennent au genre de la maxime. Ceux-ci s'adressent exclusivement au lecteur. ${ }^{7}$ Ni Mme de Raisel, ni Adélaïde ni aucun autre protagoniste du roman ne se sert manifestement de discours globalisants, ni de formules d'observations générales, ni de maximes. C'est la voix de l'auteur qui s'insinue et se glisse à leur place, interrompant de cette manière la narration pour établir un substrat moral et idéologique personnel que l'auteur cherche à rattacher objectivement à un savoir universel. Au contraire, dans les Lettres de Mylord Rivers, la situation est renversée de sorte que le discours objectif s'impose, non pas comme une réflexion libre sans appartenance particulière, mais rattaché visiblement à l'interlocuteur principal auquel on peut attribuer trois quart de ces propositions. ${ }^{8}$ Les autres réflexions se répartissent entre cinq personnages différents.

Avant d'aborder l'analyse de ces chiffres qui reflètent l'emploi des maximes, peut-être conviendrait-il d'abord de situer le féminisme de notre romancière dans une perspective historique, afin d'établir le lien qui l'unit à la pensée contemporaine sur le sujet de la femme. Le thème du mystère féminin, ${ }^{9}$ terme inventé par les hommes, a de tout temps exercé une fascination importante sur l'imaginaire universel, mais, au 18e siècle, les débats sur l'Autre' ont repris avec une force nouvelle. Avec tout le risque que comporte la simplification, disons que la pensée sur le deuxième sexe, au siècle des Lumières, se partage entre deux courants. Soulignons, au préalable, que pour les deux mouvements, la définition de la spécificité féminine était introduite de l'extérieur, fondée sur des conceptions intellectuelles de la femme et énoncée par des hommes. De nos jours, la critique dans le domaine de la femme des Lumières est encore dominée par la voix masculine qui articule deux conceptions différentes de la femme: 'l'une, ouverte sur la vie quotidienne, sur les formes concrètes du bonheur, sur les problèmes de la famille, de l'amour, souligne la particularité de la femme, estimant plus féconde, à la fois sur le plan affectif et sur le plan médical, l'idée de différences; l'autre, le courant intellectualiste (qui caractérise en particulier la philosophie du 18e siècle, prise en son sens étroit) qui est une pensée libertaire, généreuse, pour laquelle le concept d'homme est un. ${ }^{\prime 10}$ Insister sur l'égalité entre les sexes est une attitude adoptée 
par les rationalistes et les encyclopédistes. Ils dénoncent les institutions qui oppriment la femme enfermée dans son statut d'infériorité juridique et préfèrent ignorer les différences sociologiques et psychologiques entre les sexes en minimisant la portée de la différence biologique. Selon la première conception, la satisfaction de la femme viendrait de sa disposition naturelle à jouir de la vie immédiate grâce aux privilèges de son sexe, qui pencherait naturellement davantage vers la vie affective que vers la vie de l'esprit. Dans le cadre sociopolitique de l'époque, les institutions, les interdits de l'Eglise, de la famille et de l'éducation étouffent la destinée féminine sur le plan de l'intelligence. C'est dans ce courant d'orientation conservatrice et axée sur la différence féminine qu'il faut inscrire l'expérience et la pensée de Mme Riccoboni.

On pourrait, donc, voir dans Mme Riccoboni une féministe avant la lettre, et ceci de deux manières. Un peu à la façon de Mme de Lambert ${ }^{11}$ au début du siècle, elle définit la spécificité de la femme dans le cadre limité des bienséances et des valeurs sociales de l'époque. Mais elle ose se faire ouvertement, explicitement, le porte-parole des femmes qui se sentent dominées et exploitées en ce qui a trait en particulier à la vie sentimentale. Non seulement y a-t-il souvent un pacte de solidarité entre les personnages féminins de ses romans, pacte rendu manifeste par les liens d'une étroite amitié, mais mieux encore, la voix de la narratrice relaie ouvertement ses héroïnes au moyen d'observations générales et de maximes, donnant ainsi la preuve de sa solidarité avec elles. Jusqu'à présent, la critique a voulu voir dans cette protestation directe et dans le ton contestataire de ces textes un signe de faiblesse, plutôt que de nouveauté et de force, souvent parce que la revendication de l'auteur et les paroles de l'héroïne étaient confondues. Nous ne nions pas que Mme Riccoboni s'inscrive au premier abord dans la tradition conservatrice de l'époque, par sa façon timide d'envisager la personnalité de ses héroïnes souvent vues comme des individus faibles. Cette première impression est pourtant doublée d'une image plus complexe.

Dans l'Histoire de Cressy le parti-pris féministe se manifeste avec force sur deux plans. D'une part, le déroulement tragique des événements du récit symbolise déjà la pensée de l'auteur. D'autre part, la présentation de l'archétype de la féminité, qui se définit chez Mme Riccoboni par opposition à l'homme, est renforcée par l'emploi des maximes. L'essence féminine, parce que le portrait moral idéal que l'auteur trace initialement de Mme de Raisel, avec ses qualités supérieures, ne s'écarte presque jamais de cette image parfaite, si ce n'est dans la catastrophe finale dont elle est elle-même responsable: 'une physionomie ouverte annonçoit la noblesse et la candeur de son âme; la bonté, la douceur 
et la générosité formoient le fond de son caractère; incapable de feindre, elle l'étoit aussi de concevoir la plus légère défiance' (p. 3). Mme de Raisel n'est pas seulement l'épouse inimitable par sa dévotion absolue et sa constance éternelle à un mari infidèle, mais elle représente aussi l'amie idéale par sa générosité et sa bonté dans son rapport avec Adélaïde et Hortense. Du reste, cette beauté morale de Mme Raisel honore également un bon nombre d'autres héroïnes riccoboniennes à l'exclusion de sentiments et de traits à dimension plus humaine, surtout lorsqu'il s'agit de ses rapports avec les hommes. Fort heureusement ce moule de perfection est brisé au dénouement: l'heroïne devient plus intéressante par son énergie et par son humanité, ne serait-ce que par une force encore négative au moment où elle s'arrache à la stagnation de la souffrance par sa conduite résolue.

Dans les Lettres de Mylord Rivers la femme est caractérisée, comme d'ailleurs dans les autres romans de l'auteur, par une affectivité suraiguë, qui est souvent à l'origine de ses souffrances, parfois même complaisantes: 'la sensibilité est dans le coeur d'une femme la source de mille mouvemens pénibles, ${ }^{\prime 2}$ s'exclame Mme Orrery. L'accent est mis dans les deux romans sur la différence entre les sexes. L'homme, tel qu'il est envisagé par la femme compatissante, qui cherche à lui pardonner ses défauts, apparaît comme une créature plus faible qu'elle, moins capable 'de résister à l'impulsion de ses sens, d'arrêter la fougue de ses désirs. ${ }^{13}$ Il devient la marionnette de ses propres instincts qui le mènent à la séduction du libertinage.

Par contre, l'expérience de l'amour sur lequel les héroïnes riccoboniennes misent toute leur vie est une puissance dévorante. Adélaïde, la jeune innocente de seize ans et Mme de Raisel, la veuve de vingt six ans, pareilles à leurs soeurs romanesques, cherchent l'accomplissement de leur destinée en s'immolant à la passion et à l'amant. L'amour est envisagé comme un sentiment généreux et altruiste auquel l'héroïne s'abandonne sans hésitation, oubliant dans ce don d'elle-même tout sentiment de sa propre identité: 'Elle voyait un dieu dans son mari, il lui devenoit tous les jours plus cher; sans cesse occupée à lui procurer de nouveaux amusemens, elle sembloit ne vivre, ne respirer que pour répandre l'agrément sur les jours de celui qu'elle aimoit; les moindres désirs du marquis, ses plus légères fantaisies, devenoient une affaire pour madame de Cressy. Elle lui sacrifioit ses propres goûts, même le plaisir de le voir' (p. 62). Cette disposition joyeusement affectée à l'abnégation complète en faveur du bien-être de l'homme, reflète une sous-estimation évidente à l'égard de sa propre valeur. Le bonheur ne s'achète pas aux dépens de sa propre indépendance comme l'apprend Mme de Raisel, au cours de son mariage. A mesure que la déception s'introduit dans sa vie et la domine, cette héroïne humble 
mais honorable est transformée en esclave et en victime, rôle dans lequel Mme Riccoboni a tendance à enfermer ses autres héroïnes. Le refus, ou plutôt l'impossiblité de se voir en tant qu'individu libre et maître de sa vie, se conçoit comme le résultat des circonstances sociales restrictives de l'époque. Mme de Raisel se sent de plus en plus emprisonnée dans sa situation maritale. Quand son état émotionnel empire avec les infidélités de son mari, alors l'inutilité du sacrifice vient ébranler son existence.

Un changement dans l'optique de l'auteur à l'égard de la liberté féminine engendra une évolution subtile dans le type d'héroïne que présente Mme Riccoboni dans les Lettres de Mylord Rivers. Presque vingt ans après la première création d'une femme, aveugle à la valeur de sa propre vie comme Mme de Cressy et attirée par l'exil au couvent comme Adélaïde, la nouvelle héroïne Adeline refuse de se reconnaître dans le rôle de la femme mariée. ${ }^{14}$ La satisfaction intérieure obtenue à travers l'amour et le mariage n'est plus envisagée comme un but souhaitable. Dans ce dernier roman, l'union conjugale, au contraire, est considérée comme étant à l'origine d'une tranformation peu flatteuse pour la femme. Adeline Rutland est horrifiée du changement subi par sa soeur après son mariage: 'ah! bon dieu, quelle étrange métamorphose ont opérée les noeuds chers et sacrés de l'hymen ... être ... si pénétrée des devoirs de son état, si ardente à les remplir, si soumise aux loix d'un époux! ${ }^{15}$ Pour Adeline, la vie isolée de la campagne, partagée uniquement avec un mari, qui doit lui offrir tout amusement et toute occupation, ne peut lui suffire. Il est significatif, comme indice d'un discours sensiblement différent, que ce soit l'héroïne elle-même qui analyse, juge et compare la vie maritale de sa belle-soeur à la liberté qu'elle possède encore, et non pas l'auteur qui, par ailleurs, est responsable du didactisme. Adeline est plus avertie que ses prédécesseurs de ses propres désirs et aux options qu'elle peut envisager en prenant l'initiative de la direction qu'elle veut donner à sa vie.

Dans l'Histoire de Cressy, les idées sur la femme et les différences entre les sexes étaient présentées par des réflexions générales où se reconnaissait la voix de l'auteur. Ici, Mme Riccoboni prête à son héroïne une conscience lucide et une détermination fervente à résister à son sort de victime: 'je ne crois devoir à personne le sacrifice de ma liberté, ni celui de mes sentimens' (p. 289), s'exclame Adeline. Comme dans tous les domaines de la société, en cette fin de siècle, où les remous et les pressions se font sentir et où se produisent de véritables changements, ${ }^{16}$ la femme entrevoit, ne serait-ce encore que comme une étincelle fugitive, le droit à la dignité de sa liberté personnelle. Même s'il ne s'agit pour une jeune fille que de s'opposer à la domination de son frère et à celle de son tuteur, la voix intérieure lui permet enfin de satisfaire 
ses exigences personnelles par un refus catégorique de plaire aux autres plutôt qu'à elle-même. 'J'ai tout examiné, tout comparé. Il résulte de cette mûre délibération,' que l'héroïne osera combattre l'autorité des autres avec l'intention de ne plaire qu'à elle-même. ${ }^{17}$

Pour mieux saisir la profondeur du changement qui s'est opéré dans les idées de Mme Riccoboni à l'égard de la liberté personnelle, il ne reste qu'à opposer l'attitude déterminée d'Adeline en face de son sort et les réflexions rationnelles de sa belle-soeur, mylady Orrery aux sentiments de désespoir éprouvés par Mme de Cressy devant son abandon. Le dénouement surprenant et énigmatique du suicide de l'héroïne dans l'Histoire de Cressy exprimait explicitement le pessimisme de l'auteur. La résolution symbolique de l'intrigue, réalisée par la mort, était la seule solution romanesque possible pour l'auteur, à ce moment de sa vie encore entravé par les difficultés personnelles. Mme Riccoboni a utilisé le faux repos de la mort et le silencieux martyre de l'héroïne comme étant le meilleur moyen de protester pour attirer notre attention sur le sort de la femme trahie et abandonnée. Dans la société libertine du dix-huitième siècle, ce suicide inattendu a choqué le public. Vingt-cinq ans plus tard, Mme de Genlis se plaignait encore de cette fin ambiguë, mi-héroïque, mi-pathétique. Au niveau psychologique, les motifs humains d'un désespoir lucide, qui caractérisent la dernière scène du roman, résument brièvement une plainte d'une intensité émouvante. 'Rejettée d'un ingrat, trahie par celle que j'ai si tendrement recueillie, malheureuse dans ma propre maison, j'y suis l'objet de la pitié de mes valets!' (p. 107) La double déception de l'amour et de l'amitié pousse l'héroïne à sombrer dans le gouffre éternel. Faut-il y lire une liberté poussée jusqu'au nihilisme ou l'expression d'un emprisonnement absolu par les valeurs socio-culturelles?

En fait, l'interprétation du suicide n'est pas simple car l'ambiguïté foncière tient à la dualité de cet acte: le courage d'une part, la faiblesse de l'autre. La première lecture de cette mort volontaire reflèterait la rupture du rêve romantique: l'impossibilité de réaliser l'équilibre et l'harmonie parfaite dans le mariage. Ce suicide pourrait s'expliquer également comme la négation désespérée d'une vie absurde. Mais ne pourrait-on pas ajouter une autre explication en faisant de ce geste décisif de l'héroïne résolue à déterminer le cours de sa vie, le sursaut qui serait affirmation d'une liberté personnelle.

Pour une femme, au moment de la parution de ce roman, sortir de ses illusions victimisantes et changer radicalement sa destinée étaient aux yeux de tous un acte invraisemblable et inouii. Naturellement, nous ne tenons pas compte du grand nombre de femmes-auteurs qui n'en sont pas moins des exceptions en exerçant leur liberté individuelle par le fait courageux de vivre seules et indépendantes. ${ }^{18}$ Bref, on peut 
comprendre cet acte de Mme de Cressy comme l'expression métaphorique d'une promesse d'énergie, de détermination et de volonté, même si, dans son cas, le résultat s'avère négatif. Ce dénouement peut annoncer la possibilité qui existe de s'évader de la médiocrité et de l'insatisfaction quand l'action vise à atteindre un but positif.

Au bout de sa carrière littéraire, dans les Lettres de Mylord Rivers, Mme Riccoboni transforme cette force, jadis négative, grâce à l'expérience personnelle, en une vague libératrice. Adeline, à la veille de la Révolution, aurait pu être un modèle de femme des Lumières, certes dans un sens encore limité, mais modèle d'inspiration tout de même. En conclusion, la narration à la troisième personne, Histoire de $M$. de Cressy illustre les déceptions associées à une vision romantique du mariage fondée sur l'idéalisme et l'abnégation. Par contre, le dernier roman épistolaire de l'auteur, Les Lettre de Mylord Rivers, affirmant une maîtrise de soi, reflète la mimésis d'une optique stoïque dans l'action.

\author{
OLGA B. CRAGG
}

University of British Columbia

\title{
Notes
}

1 Nous nous rendons compte que cette expression, employée de plus en plus fréquemment, risque d'offenser la sensibilité linguistique des puristes. Le terme 'féministe' est d'usage relativement récent, remontant seulement à 1841.

2 Tout récemment deux ouvrages importants ont paru sur la rhétorique, l'art de la persuasion dans le roman du 18e siècle: Jean-Paul Sermain, Rhétorique et roman au dix-huitième siecle. L'exemple de Prévost et de Marivaux (1728-1742), Studies on Voltaire and the Eighteenth Century, 233 (Oxford: The Alden Press, 1985). La seconde étude se penche sur les questions de méta-langage associées au désir de convaincre par l'emploi du discours sentencieux qui se veut règle ou loi. Geoffrey Bennington, Sententiousness and the Novel. Laying Down the Law in Eighteenth-Century French Fiction (Cambridge: Cambridge UP, 1985).

3 Ce titre a été souvent raccourci à l'Histoire du marquis de Cressy à partir de 1773. Dans notre étude nous allons renvoyer au roman sous la forme abrégée $\mathrm{d}^{\prime}$ Histoire de Cressy.

4 Marie-Jeanne Riccoboni, Mme Riccoboni's letters to David Hume, David Garrick and Sir Robert Liston: 1764-1783, ed. James C. Nicholls, Studies on Voltaire and the Eighteenth Century, 159 (Banbury, Oxfordshire: Cheney and Sons, 1976), 266.

5 Marie-Jeanne Riccoboni, Oeuvres complètes, (Paris: Volland, 1786). C'est à cette édition que renvoient les références que nous donnons dans le texte après les citations. 
6 Voir la classification, la terminologie et la fonction des vérités générales selon F. Rodegem dans 'La Parole proverbiale,' Richesse du proverbe, eds. François Suard et Claude Buridant, II (Lille: Presses de l'Université de Lille III, 1984), 121-135.

7 Nous sommes redevables à Peter France pour Racine's rhetoric (Oxford: Oxford UP, 1965) et à Jean-Paul Sermain de la distinction qu'ils apportent à ces deux degrés de rhétorique, l'un qui appartient au niveau de l'histoire (sens genettien, Gérard Genette Figures III, (Paris: éditions du Seuil, 1972), 72 et l'autre au dialogue entre créateur-public-lecteur. Sermain, Rhétorique, 2.

8 A titre d'exemple, comparons le nombre de maximes dans l'Histoire de Cressy et dans les Lettres de Mylord Rivers à celui du Paysan parvenu et de La Vie de Marianne.

$\begin{array}{lrrrr} & \begin{array}{r}\text { La Vie } \\ \text { de Marianne }\end{array} & \begin{array}{r}\text { Le Paysan } \\ \text { Parvenu }\end{array} & \begin{array}{r}\text { Histoire } \\ \text { de Cressy }\end{array} & \begin{array}{r}\text { Lettres de } \\ \text { Mylord Rivers }\end{array} \\ \begin{array}{l}\text { Nombre de } \\ \text { lignes }\end{array} & 19,593 & 9,025 & 3,220 & 6,297 \\ \text { Mots par ligne } & 11,8 & 11,8 & 8 & 8 \\ \text { Total de mots } & 231,197 & 106,459 & 25,760 & 50,376 \\ \text { Nombre de } & 166 & 117 & 43 & 61 \\ \text { parémies } & \text { soit } 7.2 & 10.9 & 16.7 & 12.1 \\ \begin{array}{l}\text { Intervalle de } \\ \text { mots entre les }\end{array} & & & & \\ \text { parémies } & 1,392 \text { mots } & 910 \text { mots } & 599 \text { mots } & 825 \text { mots }\end{array}$

On voit clairement par ce schéma que la fréquence des parémies est la plus grande chez Mme Riccoboni. On peut en déduire que dans les Lettres de Mylord Rivers, il y a 29 pourcent moins de maximes que dans l'Histoire de Cressy.

9 Diderot lui-même revient à cette idée de la nature cachée de la femme dans son essai 'Sur les femmes,' Oeuvres (Paris: Gallimard, 1951), 957.

10 Paul Hoffmann, La Femme dans la pensée des Lumières (Paris: Ophrys, 1977), 562.

11 Jeannette Geffriaud Rosso, Etudes sur la féminité aux XVIIe et XVIIIe siècles (Pisa: Libreria Goliardica, 1984), 77-89.

12 Marie Jeanne Riccoboni, Lettres de Mylord Rivers (Paris: Volland, 1786), 470.

13 Marie Jeanne Riccoboni, Lettres de Mylord Rivers, 275. La thématique de la violence des passions est soulignée encore une fois à la fin du roman où Rivers justifie l'impétuosité des sentiments par un argument basé sur l'habitude. 'Dans le coeur d'une femme réservée et délicate, l'amour peut être une passion douce, il peut occuper son âme sans la troubler; l'attendrir sans l'écarter des bornes de la modération et des règles de la décence. Mais cette même passion agite, tourmente un sexe plus libre, plus hardi, moins accoutumé à maîtriser ses sens: elle se change dans son sein en une ardeur pénible, il souffre de l'impétuosité de ses désirs, et leur violence lui impose la nécessité de les satisfaire ou celle de les éteindre': Lettres de Mylord Rivers, 409.

14 Remarquons qu'une des comparses de ce roman, mylady Orrery, est une veuve qui sait apprécier les avantages de son état. La liberté, la paix et l'indépendance qu'elle goûte depuis onze ans sont les biens qu'elle évoque au moment où elle risque de succomber à la tentation de l'amour. 
15 Riccoboni, Lettres de Mylord Rivers, 297-98.

16 Le côté documentaire des Lettres de Mylord Rivers reflète justement cette participation plus vigoureuse des femmes dans la vie de la société: 'Tout le monde projette, tout le monde établit des principes, tout le monde forme des plans d'administration. Les femmes même s'occupent de ces graves objets. L'esprit de parti s'introduit à la toilette, siège à table, se mêle à tous les jeux. Une jeune beauté choisit et protège un système politique, proscrit les autres, dispute, et quelquefois s'emporte,' 286.

17 Riccoboni, Lettres de Mylord Rivers, 298.

18 Souvenons-nous des onze célibataires sur 25 noms retenus entre 1740 et 1780 qui ont choisi une vie libre. Marie-Laure Swiderski, 'La Condition de la femme française au 18e siècle,' présentation orale au Septième congrès international des Lumières, Budapest, août, 1987. 